\title{
Viscosity Solutions of the Bellman Equation for Perturbed Optimal Control Problems with Exit Times ${ }^{0}$
}

\author{
Michael Malisoff \\ Department of Mathematics \\ Louisiana State University \\ Baton Rouge, LA 70803-4918 USA \\ malisoff@math.lsu.edu
}

\begin{abstract}
In a series of papers, we presented new theorems characterizing the value function in optimal control as the unique bounded-from-below viscosity solution of the corresponding Bellman equation that satisfies appropriate side conditions. Instead of the usual assumption that the instantaneous costs are uniformly positive, our results assumed that all trajectories satisfying a certain integral condition must asymptotically approach the target. In this note, we study perturbed exit time problems which have the property that all trajectories satisfying the integral condition must stay in a bounded set. This is a weaker asymptotic property, since it allows bounded oscillating trajectories and attractors other than the target. We show that, under this weaker asymptotic condition, the value function is still the unique bounded-from-below solution of the corresponding Bellman equation that vanishes on the target. Our theorem applies to problems which are not tractable by the known results. The significance of our work is that (i) applied control abounds with problems whose dynamics are only known up to a margin of error, which can be represented by perturbations, and (ii) our theorem implies the convergence of numerical methods which can be used to approximate value functions for problems that satisfy our relaxed hypotheses.
\end{abstract}

\section{Introduction}

Control theory abounds with challenging situations which can be studied by means of the (HamiltonJacobi-)Bellman equation, i.e., the HJBE (cf. [1,

\footnotetext{
${ }^{0}$ August 19, 2002 Draft. Some of this work was carried out during the spring of 2000 , while the author was a Research Associate at Washington University in St. Louis and supported by DARPA-JFACC Grant F30602-99-2-0551.
}

4]). A fundamental issue in the analysis of Bellman equations is uniqueness of solutions of the equation subject to appropriate boundary conditions. Starting from uniqueness results of this kind, it is possible to estimate the rate of convergence of numerical schemes for approximating minimum cost functions, and to study singular perturbations and much more (cf. the papers $[2,4,5,7]$ for uniqueness of HJBE solutions, and [1, 3] for the numerical analysis applications). ${ }^{1}$

This note will extend the results of [4] on proper HJBE solutions by developing uniqueness theory for bounded-from-below solutions ${ }^{2}$ of

$$
\sup _{a \in A}\{-f(x, a) \cdot D w(x)-\ell(x, a)\}=0, x \notin \mathcal{T}
$$

which is the HJBE corresponding to the (undiscounted) exit time problem

$$
\begin{aligned}
& \text { Infimize } \int_{0}^{t_{x}(\beta)} \ell^{r}\left(y_{x}(s, \beta), \beta(s)\right) d s \\
& \text { over all } \beta \in \mathcal{A} \text { for which } t_{x}(\beta)<\infty
\end{aligned}
$$

for all $x \in \mathbb{R}^{N}$, where $y_{x}(\cdot, \beta)$ is the solution of

$$
\begin{aligned}
& \frac{d}{d s} y_{x}(s, \beta)=f^{r}\left(y_{x}(s, \beta), \beta(s)\right) \text { a.e. } \\
& y_{x}(0, \beta)=x, \quad \beta \in \mathcal{A}, \quad x \in \mathbb{R}^{N}
\end{aligned}
$$

In (2)-(3), $A \subset \mathbb{R}^{N}$ is a fixed nonempty compact set (called the control set), $\mathcal{T} \subseteq \mathbb{R}^{N}$ is a fixed closed, nonempty, possibly unbounded set (called the target $), t_{x}(\beta):=\inf \left\{t \geq 0: y_{x}(t, \beta) \in \mathcal{T}\right\}$ (which we refer to as an exit time), and $\mathcal{A}$ (which is called the set of relaxed controls) is the set of all measurable functions $\alpha:[0, \infty) \rightarrow A^{r}$, where

\footnotetext{
${ }^{1}$ See also the companion paper [6] to this note, which applies these results to stability and Lyapunov functions.

${ }^{2}$ A function $w: \mathbb{R}^{N} \rightarrow \mathbb{R}$ is bounded-from-below provided there is a finite constant $b$ such that $w(x) \geq b$ for all $x \in \mathbb{R}^{N}$. This is less restrictive than properness of $w$, which is the condition $w(x) \rightarrow+\infty$ as $\|x\| \rightarrow+\infty$.
} 
$A^{r}$ is the set of all Radon probability measures on $A$ topologized as a subset of the dual of $C(A)=$ $\{$ continuous functions $A \rightarrow \mathbb{R}\}$ with the weak- $\star$ topology (cf. [4]). We will refer to $f$ as the dynamics, $\ell$ will be called the instantaneous cost (a.k.a. Lagrangian), and $h^{r}(x, m):=\int_{A} h(x, a) d m(a)$ for $h=f$ or $h=\ell, x \in \mathbb{R}^{N}$ and $m \in A^{r}$. (If $\{(f(x, a), \ell(x, a)): a \in A\}$ is convex for all $x \in \mathbb{R}^{N}$, then all of our results remain true if $\mathcal{A}$ is replaced by its subset $\{$ measurable functions $[0, \infty) \rightarrow A\}$, by the Filippov selection theorem.) Let $\|\cdot\|$ denote the usual Euclidean norm. We always assume

$\left(A_{1}\right) f: \mathbb{R}^{N} \times A \rightarrow \mathbb{R}^{N}$ is continuous, $\exists L>0$ for which $\|f(x, a)-f(y, a)\| \leq L\|x-y\|$ for all $x, y \in \mathbb{R}^{N}$ and $a \in A$.

$\left(A_{2}\right) \ell: \mathbb{R}^{N} \times A \rightarrow[0, \infty)$ is continuous.

In particular, we do not assume uniform positive lower bounds on $\ell$, and $\ell$ need not be locally Lipschitz, so the usual uniqueness results for (1) from $[1,9]$ do not apply. Exit time problems of this kind have been extensively analyzed (cf. [1, 2, 4, 7, 9], and $\S 2$ for how these works relate to the uniqueness result we prove in this note). In particular, the papers $[7,9]$ studied the case where the following strong asymptotic condition is satisfied:

$$
\begin{aligned}
& \int_{0}^{\infty} \ell^{r}\left(y_{x}(s, \alpha), \alpha(s)\right) d s<\infty \Rightarrow \\
& \lim _{s \rightarrow+\infty} y_{x}(s, \alpha) \in \mathcal{T}
\end{aligned}
$$

For any closed set $B \subseteq \mathbb{R}^{N}, S T C_{f}(B)$ is the condition that for each $\varepsilon>0, B \subseteq \operatorname{int}\left(\mathcal{R}_{B}^{\varepsilon}\right)$, where $\mathcal{R}_{B}^{\varepsilon}:=$ $\left\{x \in \mathbb{R}^{N}: \exists \alpha \in \mathcal{A} \& t \in[0, \varepsilon)\right.$ s.t. $\left.y_{x}(t, \alpha) \in B\right\}$. We sometimes write $y_{x}^{f}(\cdot, \alpha)$ to mean $y_{x}(\cdot, \alpha)$, to emphasize the dynamics $f$ used in $(3)$, and $\partial(B)$ denotes the boundary of any set $B \subseteq \mathbb{R}^{N}$. Also, $\operatorname{dist}(x, B):=\inf \{\|x-b\|: b \in B\}$ for all $B \subseteq \mathbb{R}^{N}$.

In (2)-(3), the data $f, \ell$, and $\mathcal{T}$ are known with certainty, and the goal is to establish that the (exit time) value function (a.k.a. minimal cost function) $v: \mathbb{R}^{N} \rightarrow \mathbb{R} \cup\{+\infty\}$ for these data, which is defined by

$v(x)=\inf _{\left\{\alpha \in \mathcal{A}: t_{x}(\alpha)<\infty\right\}} \int_{0}^{t_{x}(\alpha)} \ell^{r}\left(y_{x}^{f}(s, \alpha), \alpha(s)\right) d s$,

is the unique bounded-from-below solution of (1) that is null on the target $\mathcal{T}$. Since $v$ will not in general be differentiable, we will view our solutions in the sense of viscosity solutions, by which we mean the following. Let $C^{1}(\mathcal{G})$ denote the set of all real-valued continuous functions on any open set $\mathcal{G} \subseteq \mathbb{R}^{N}$ which have one continuous derivative, and let $C(\mathcal{G})$ denote the set of all continuous functions $\mathcal{G} \rightarrow \mathbb{R}$. Also, $C^{1}$ alone means $C^{1}\left(\mathbb{R}^{N}\right)$, and $B_{r}(q):=\left\{p \in \mathbb{R}^{N}:\|p-q\|<r\right\}$ for all $r>0$ and $q \in \mathbb{R}^{N}$. For each locally bounded function $w: S \rightarrow \mathbb{R}$ on a set $S \subseteq \mathbb{R}^{N}$, we define

$$
w_{\star}(x):=\liminf _{S \ni y \rightarrow x} w(y), \quad w^{\star}(x):=\limsup _{S \ni y \rightarrow x} w(y) .
$$

Note that $w \equiv w^{\star} \equiv w_{\star}$ if $w$ is continuous.

Definition 1.1 Assume $\mathcal{G} \subseteq \mathbb{R}^{N}$ is open, $S \supseteq \mathcal{G}$, $F: \mathbb{R}^{N} \times \mathbb{R}^{N} \rightarrow \mathbb{R}$ is continuous, and $w: S \rightarrow \mathbb{R}$ is locally bounded. We call $w$ a (viscosity) solution of $F(x, D w(x))=0$ on $\mathcal{G}$ provided: If $\gamma \in C^{1}(\mathcal{G})$ and $x_{o} \in \mathcal{G}$ is a local minimizer (resp., maximizer) of $w_{\star}-\gamma\left(\right.$ resp., $\left.w^{\star}-\gamma\right)$, then $F\left(x_{o}, D \gamma\left(x_{o}\right)\right) \geq 0$ (resp., $\left.F\left(x_{o}, D \gamma\left(x_{o}\right)\right) \leq 0\right)$.

From the point of view of applied control, uniqueness results for HJBE solutions are of great interest, because they imply the convergence of methods for approximating the value function, convergence of singular perturbations, and much more (cf. [1, 3]).

However, in real-life control applications, it is often the case that the data $f, \ell$, and $\mathcal{T}$ are not known with certainty. Instead, approximate formulas (i.e., "educated guesses") $f=f_{0}, \ell=\ell_{0}$, and $\mathcal{T}=\mathcal{T}_{0}$ for these data are known from experiments or field observations, and then control theory can be applied to the problems whose dynamics, Lagrangians, and targets are given respectively by

$$
\begin{aligned}
& f_{p}(x, a)=f_{o}(x, a)+\phi_{f}(p, x, a), \\
& \ell_{p}(x, a)=\ell_{o}(x, a)+\phi_{\ell}(p, x, a), \\
& \mathcal{T}_{p}=\mathcal{T}_{o}+\phi_{\mathcal{T}}(p)
\end{aligned}
$$

for various values of the parameter $p \geq 0$, where the functions $\phi_{f}, \phi_{\ell}$, and $\phi_{\mathcal{T}}$ (representing perturbations) are null for $p=0$ and satisfy $\sup \left\{\left\|\phi_{f}(p, x, a)\right\|+\left|\phi_{\ell}(p, x, a)\right|+\|q\|:(x, a) \in\right.$ $\left.\mathbb{R}^{N} \times A, q \in \phi_{\mathcal{T}}(p)\right\} \rightarrow 0$ as $p \rightarrow 0$. Often, information is known about the perturbation functions, but the true value of $p$ is not known. For example, the parameter $p$ could represent the possible objectives of the enemy in military operations. In some cases, the unperturbed data $f=f_{0}, \ell=\ell_{0}$, and $\mathcal{T}=\mathcal{T}_{0}$ satisfy (4) and $S T C_{f_{o}}\left(\mathcal{T}_{o}\right)$, so the methods of [7] establish that the corresponding value function (5) is the unique bounded-from-below solution of the Bellman equation (1) that vanishes on $\mathcal{T}_{o}$. On the other hand, (4) and $S T C_{f}(\mathcal{T})$ may no longer hold if the data is perturbed to $f=f_{p}, \ell=\ell_{p}, \mathcal{T}=\mathcal{T}_{p}$, even if $p$ is near zero (e.g., take $\phi_{f} \equiv 0, \phi_{\ell} \equiv 0$, any nonzero singleton $\mathcal{T}=\{q\}$, and $\left.\phi_{\mathcal{T}}(p)=\{p q\}\right)$. In 
this case, it may not be possible to say that the value function $v_{p}$ for the exit problem corresponding to the data $f=f_{p}, \ell=\ell_{p}, \mathcal{T}=\mathcal{T}_{p}$ in (6) is the unique solution of the associated HJBE, so numerical methods for approximations of $v_{p}$ may not be available. One might expect in this situation that if (i) support $\left(\phi_{f}(p, \cdot)\right) \subseteq \mathbb{R}^{N} \times A$ is bounded for each $p$, (ii) $\mathcal{T}_{o}$ is bounded, and (iii) $\ell_{p} \equiv \ell_{o}$ on $\mathbb{R}^{N} \times \partial(A)$ for all $p$, then the perturbed data (6) instead satisfy the weaker condition

$$
\begin{aligned}
& {\left[\int_{0}^{\infty} \ell_{p}^{r}\left(y_{x}^{f_{p}}(s, \alpha), \alpha(s)\right) d s<\infty \Rightarrow\right.} \\
& \left.\sup \left\{\left\|y_{x}^{f_{p}}(s, \alpha)\right\|: s \geq 0\right\}<\infty\right] \forall p \geq 0
\end{aligned}
$$

Here is a motivating example from [5], called the Shifted Fuller Example, where this occurs:

Example 1.2 We take $N=2$, and for each $p \geq 0$, consider the exit time problem (2) whose target, control set, dynamics, and Lagrangian are given by

$$
\begin{aligned}
& \mathcal{T}_{p}=\{(p, p)\}, A=[-1,+1], \\
& f_{p}(x, a)=\left(x_{2}-p \Phi(x, p), a\right), \\
& \ell_{p}(x, a)=x_{1}^{2}+p(1-|a|)^{2},
\end{aligned}
$$

respectively, where for each $p \geq 0, \Phi(\cdot, p): \mathbb{R}^{2} \rightarrow$ $[0,1]$ is a $C^{1}$ function which is 1 on $B_{p / 4}((p, p))$ and 0 on $\mathbb{R}^{2}-B_{p / 2}((p, p))$. This is a perturbed version of the Fuller Example (FE), which is the case where $p=0$ in (7). Recall (cf. [8]) that the FE admits an optimal control $\beta_{z}$ for each initial state $z \in \mathbb{R}^{2}$, which is defined as follows. Set $\zeta:=\left\{\left(x_{1}, x_{2}\right):\left|x_{1}\right|=C x_{2}^{2}, x_{1} x_{2} \leq 0\right\} \subset \mathbb{R}^{2}$, set $\zeta^{ \pm}=\left\{\left(x_{1}, x_{2}\right) \in \zeta: \pm x_{1}>0\right\}$, and let $A^{-}$and $A^{+}$ denote the regions lying above and below $\zeta$ respectively, where $C>0$ is the constant root specified in [8]. Define the feedback $k: \mathbb{R}^{2} \rightarrow[-1,+1]$ by $k(q)=-1$ if $q \in A^{-} \cup \zeta^{-}, k(q)=1$ if $q \in A^{+} \cup \zeta^{+}$, and $k(0,0)=0$, and let $\gamma_{z}$ be the closed-loop trajectory for the feedback $k$ starting at $z$. We then take $\beta_{z}(t)=+1$ if $\gamma_{z}(t) \in A^{+}, \beta_{z}(t)=-1$ if $\gamma_{z}(t) \in A^{-}$, and $\beta_{z}(t)=0$ if $\gamma_{z}(t)=(0,0)$. Let $v_{p}$ denote the value function (5) for the perturbed data $f=f_{p}, \ell=\ell_{p}, \mathcal{T}=\mathcal{T}_{p}$, and $A$, as defined in (7).

As shown in [7], $v_{o}$ is the unique bounded-frombelow solution of the corresponding HJBE in the class of all continuous functions $w: \mathbb{R}^{2} \rightarrow \mathbb{R}$ which are null at $(0,0)$. This follows because the FE data satisfy (4). On the other hand, (4) no longer holds if the data is perturbed to (7) for $p>0$.

To see why (4) fails for $p>0$, set $y_{q}^{p}(\cdot, \alpha) \equiv$ $y_{q}^{f_{p}}(\cdot, \alpha)$ in what follows. For $n \in \mathbb{N}$ and $\beta_{z}$ as above, let $q(n):=\left(1 /\left(2 n^{2}\right), 1 / n\right)=y_{(0,0)}^{o}(1 / n, \alpha \equiv$
1) and $t_{n}:=\inf \left\{t \geq 0: y_{q(n)}^{o}\left(t, \beta_{q(n)}\right)=(0,0)\right\}$. Using [8], we have $M:=\sup \left\{t_{n}: n \in \mathbb{N}\right\}<\infty$. Let $\beta$ be the concatenation of $\beta_{q(1)}\left\lceil\left[0, t_{1}\right]\right.$ followed by $\alpha \equiv 1\left\lceil[0,1 / 2]\right.$ followed by $\beta_{q(2)}\left\lceil\left[0, t_{2}\right]\right.$ followed by $\alpha \equiv 1\left\lceil[0,1 / 3]\right.$ followed by $\beta_{q(3)}\left\lceil\left[0, t_{3}\right]\right.$ followed by $\alpha \equiv 1[[0,1 / 4]$ and so on. Then $|\beta| \equiv 1$. (If the input $\beta$ is used for the initial position $q(1)$ and $f_{o}$, then the resulting trajectory runs to 0 along the optimal FE trajectory for the initial value $q(1)$, then goes to $q(2)$ using $\alpha \equiv 1$, then takes the optimal FE trajectory to the origin, then uses $\alpha \equiv 1$ to go to $q(3)$, etc..) Since the norm of the first coordinate of $y_{q(n)}^{o}\left(\cdot, \beta_{q(n)}\right)$ is always below $1 / n^{2}$ (cf. [8]), $v_{o}(q(n)) \leq M / n^{4}$ for all $n$. For all $n \geq 2$, set

$\tilde{t}_{n}=\sum_{j=1}^{n-1}\left[t_{j}+(j+1)^{-1}\right] \quad$ and $\quad \gamma_{n}(s)=\beta\left(s+\tilde{t}_{n}\right)$, so $q(n)=y_{q(1)}^{o}\left(\tilde{t}_{n}, \beta\right)$. For each $p \geq 0$, there is a neighborhood $S_{p}$ of the origin such that $f_{p} \equiv f_{o}$ and $\ell_{p} \equiv \ell_{o}$ on $S_{p} \times\{ \pm 1\}$. Therefore, each $p>0$ admits an $n(p) \in \mathbb{N}$ such that, for all $s \geq 0$,

$$
y_{q(n(p))}^{o}\left(s, \gamma_{n(p)}\right)=y_{q(n(p))}^{p}\left(s, \gamma_{n(p)}\right),
$$

so

$$
\int_{0}^{\infty} \ell_{p}\left(y_{q(n(p))}^{p}\left(s, \gamma_{n(p)}\right), \gamma_{n(p)}(s)\right) d s<\infty
$$

follows from an elementary calculation (cf. [5]), even though $\lim _{s \rightarrow+\infty} y_{q(n(p))}^{p}\left(s, \gamma_{n(p)}\right)=0 \notin \mathcal{T}_{p}$.

In Example 1.2, condition (4) holds for $f=f_{p}$, $\ell=\ell_{p}$, and $\mathcal{T}=\mathcal{T}_{p}$ when the parameter $p=0$, but not if $p>0$. Moreover, the perturbed data gave HJBEs which were not tractable by the known uniqueness results for bounded-from-below solutions. However, one can show (cf. §4) that for all $p \geq 0$, the relaxed asymptotic condition

$$
\begin{aligned}
& \int_{0}^{\infty} \ell^{r}\left(y_{x}^{f}(s, \alpha), \alpha(s)\right) d s<\infty \Rightarrow \\
& \sup _{s \geq 0}\left\|y_{x}(s, \alpha)\right\|<\infty
\end{aligned}
$$

holds for the perturbed data $f=f_{p}, \ell=\ell_{p}$ in (7). As discussed above, it is important to be able to show that value functions for optimal control problems uniquely solve the corresponding HJBEs (subject to appropriate side conditions). Also, Example 1.2 illustrates how (8) can be more stable with respect to perturbations. Moreover, condition (8) does not depend on the target. This motivates our new uniqueness theorem for solutions of (1), under the relaxed condition (8), instead of the more restrictive condition (4). This theorem will be announced in $\S 2$. We discuss the proof of our theorem in $\S 3$. In $\S 4$, we apply our result to the Shifted Fuller Example. 


\section{Announcement of New Uniqueness Characterization}

We use the following definition.

Definition 2.1 We say that a function $w: \mathbb{R}^{N} \rightarrow$ $\mathbb{R}$ is weakly bounded-from-below, and write $w \in W B B$, provided there exists a sequence of bounded open sets $B_{j} \subseteq \mathbb{R}^{N}$ satisfying:

(1) $\overline{B_{j}} \subset B_{j+1}$ for all $j$, and $\mathbb{R}^{N}=\cup_{j} B_{j}$

(2) $\mathcal{L}:=\inf \left\{w(x): x \in \partial\left(B_{j}\right), j \in \mathbb{N}\right\}>-\infty$

(3) $\lim _{j \rightarrow+\infty} \min \left\{\|x\|: x \in \partial\left(B_{j}\right)\right\}=+\infty$.

Note that $w \in W B B$ for any bounded-from-below function $w: \mathbb{R}^{N} \rightarrow \mathbb{R}$. However, note that functions $w \in W B B$ need not be bounded-from-below. In $\S 3$, we will sketch the proof of the following:

\section{Theorem 1 Assume the following:}

1) $\mathcal{T} \subseteq \mathbb{R}^{N}$ is closed and nonempty.

2) $f$ and $\ell$ satisfy $\left(A_{1}\right)-\left(A_{2}\right), S T C_{f}(\mathcal{T})$, and (8).

3) If $t \in(0, \infty), \alpha \in \mathcal{A}$, and $x \in \mathbb{R}^{N} \backslash \mathcal{T}$, then $\int_{0}^{t} \ell^{r}\left(y_{x}(s, \alpha), \alpha(s)\right) d s>0$.

4) $w \in W B B$ is a solution of (1), $w$ is null and continuous at each point in $\mathcal{T}, w_{\star} \in C\left(\mathbb{R}^{N}\right)$.

Then $w \equiv v$ on $\mathbb{R}^{N}$.

Remark 2.2 Under hypotheses 1)-2) of Theorem 1 , if $v$ is finite and continuous on $\mathbb{R}^{N}$, then $v$ is a solution of (1) (cf. [1]). In that case, $v$ is the unique solution of (1) in the class of all continuous functions $w \in W B B$ which are null on $\mathcal{T}$, by Theorem 1. For extensions to solutions of (1) on general open sets and unbounded $A$, see [5]. We use the continuity of $w_{\star}$ to guarantee that the sublevel sets $\left\{x \in \mathbb{R}^{N}: w_{\star}(x)<\kappa\right\}, \kappa>0$, are open.

Remark 2.3 Theorem 1 applies to uniqueness problems for HJBEs which are not tractable by the standard results or using $[4,7]$. For example, $[1,2]$ require the following: For each $\varepsilon>0$, there exists a constant $C_{\varepsilon}>0$ such that

$$
[\operatorname{dist}(x, \mathcal{T})>\varepsilon] \Rightarrow\left[\ell(x, a) \geq C_{\varepsilon} \forall a \in A\right] .
$$

On the other hand, our conditions allow $\inf _{a} \ell(\cdot, a)$ to be null at points outside $\mathcal{T}$. They also allow cases where $\inf _{a} \ell(x, a) \rightarrow 0$ as $\|x\| \rightarrow+\infty$, which are also not allowed under (9) when $\mathcal{T}$ is bounded. In fact, as shown by example in $[4,5]$, the usual uniqueness results for HJBE solutions are no longer true if assumption (9) is omitted. The results of [4] apply to exit time problems violating (9), and give conditions guaranteeing that $v$ is the unique solution of the corresponding HJBE in a certain class of functions which are proper. The results of [4] require condition 3 ) from Theorem 1 , but they do not require (8). In [7], uniqueness results are given for problems which violate (9) but which do satisfy (4). On the other hand, Theorem 1 applies to problems violating both (4) and (9). While the results of [4] apply to cases where neither (4) nor (9) holds, the conclusions of those results are that if the value function is proper, then it is the unique proper solution of the HJBE satisfying appropriate side conditions. For results on nonnegative solutions of (1) for locally Lipschitz $\ell$, see [9]. Since we do not need to assume properness or nonnegativity of solutions in Theorem 1, our results improve $[4,9]$ for cases where (8) is satisfied. Condition (4) has the 'optimistic' interpretation that if a feasible trajectory is followed on $[0, \infty)$, then it must eventually 'find' the target $\mathcal{T}$, at least asymptotically. Condition (8) has the more 'conservative' economic interpretation that if a path can be followed on $[0, \infty)$ at finite cost, then there is some finite bound on how far points along the path can ever get from the starting point. Also, (8) is less restrictive than (4) when $\mathcal{T}$ is bounded, since it allows oscillating trajectories and attractors outside $\mathcal{T}$.

\section{Sketch of Proof of Uniqueness Characterization}

In this section, we will sketch the proof of Theorem 1. For a detailed proof for the special case where $B_{j} \equiv B_{j}(0)$, see [5]. We assume that $w$ is continuous, the proof of the general case being similar.

The proof that $w \leq v$ is a special case of the proof of Proposition 5.4 in [4]. It remains to show that $w \geq v$. We omit the superscripts $r$ to simplify notation. Let $\left\{B_{j}\right\}$ be as in Definition 2.1. Fix $x \in \mathbb{R}^{N} \backslash \mathcal{T}, \kappa>w(x)$, and $J \in \mathbb{N}$ for which $x \in B_{J}$. Set $S_{\kappa}=\left\{x \in \mathbb{R}^{N}: w(x)<\kappa\right\}$, which is open because $w$ is continuous. Set $\mathcal{S}=S_{\kappa} \cap B_{J}$. Set $\tau_{p}(\beta):=\inf \left\{t \geq 0: y_{p}(t, \beta) \in \partial(\mathcal{S} \backslash \mathcal{T})\right\}$ for each $p \in \mathbb{R}^{N}$ and $\beta \in \mathcal{A}$. Fix $\left.\varepsilon \in\right] 0, \kappa-w(x)[$. Set

$I(x, t, \alpha):=\int_{0}^{t} \ell\left(y_{x}(s, \alpha), \alpha(s)\right) d s+w\left(y_{x}(t, \alpha)\right)$ 
wherever the RHS is defined. We also set

$$
T_{\delta}(p)=\inf _{\alpha \in \mathcal{A}}\left\{t \geq 0: \operatorname{dist}\left(y_{p}(t, \alpha), \partial(\mathcal{S} \backslash \mathcal{T})\right)<\delta\right\}
$$

for all $p \in \mathbb{R}^{N}$ and $\delta>0$, and we define $x_{1}:=x$, $\tau_{1}:=T_{1}\left(x_{1}\right)$ when $T_{1}\left(x_{1}\right)<+\infty$, and $\tau_{1}:=10$ when $T_{1}\left(x_{1}\right)=+\infty$. We can then use the representation lemma (cf. [5]) to find a relaxed control $\alpha_{1} \in \mathcal{A}$ such that $w\left(x_{1}\right) \geq I\left(x_{1}, \tau_{1}, \alpha_{1}\right)-\varepsilon / 4$. Note that $y_{x_{1}}\left(\tau_{1}, \alpha_{1}\right) \in \mathcal{S} \backslash \mathcal{T}$. By induction, we can now define a sequence

$$
x_{k}:=y_{x_{k-1}}\left(\tau_{k-1}, \alpha_{k-1}\right) \in \mathcal{S} \backslash \mathcal{T}
$$

for $k=2,3, \ldots$, where

$$
\tau_{k}:= \begin{cases}T_{1 / k}\left(x_{k}\right) & \text { if } T_{1 / k}\left(x_{k}\right)<+\infty \\ 10^{k} & \text { otherwise }\end{cases}
$$

and the controls $\alpha_{k} \in \mathcal{A}$ are chosen (again using the representation lemma) to satisfy

$$
w\left(x_{k}\right) \geq I\left(x_{k}, \tau_{k}, \alpha_{k}\right)-2^{-(k+1)} \varepsilon \forall k .
$$

We also define $\sigma_{o}=0, \sigma_{k}:=\tau_{1}+\ldots+\tau_{k}, \bar{\sigma}_{J}=$ $\lim \sup _{k} \sigma_{k}$, and, for an arbitrary $\bar{a} \in A$,

$$
\bar{\alpha}_{J}(s):= \begin{cases}\alpha_{1}(s) & \text { if } 0 \leq s<\sigma_{1}, \\ \alpha_{2}\left(s-\sigma_{1}\right) & \text { if } \sigma_{1} \leq s<\sigma_{2}, \\ \vdots & \\ \alpha_{k}\left(s-\sigma_{k-1}\right) & \text { if } \sigma_{k-1} \leq s<\sigma_{k}, \\ \vdots & \\ \bar{a} & \text { if } \bar{\sigma}_{J} \leq s,\end{cases}
$$

with the last line used if $\bar{\sigma}_{J}<+\infty$. Reapplying (11) and summing on $k \in \mathbb{N}$, we therefore get

$$
w(x) \geq I\left(x, \sigma_{k}, \bar{\alpha}_{J}\right)-\frac{\varepsilon}{2}\left(1-\frac{1}{2^{k}}\right) \quad \forall k \in \mathbb{N} .
$$

By (10) and the boundedness of $\mathcal{S}$, we can find $\bar{x}_{J} \in$ $\overline{\mathcal{S}}$ and a subsequence (which we will not relabel) for which $x_{n} \rightarrow \bar{x}_{J}$. In fact, a variant of an argument from [4] allows us to use hypothesis 3) to conclude that $\bar{x}_{J} \in \partial(\mathcal{S} \backslash \mathcal{T})$ (cf. [5] for full details). Since

$$
\partial(\mathcal{S} \backslash \mathcal{T}) \subseteq \partial\left(\mathcal{S}_{\kappa}\right) \cup \mathcal{T} \cup \partial\left(B_{J}\right),
$$

we have the following cases to consider:

Case 1: If $\bar{x}_{J} \in \partial\left(\mathcal{S}_{\kappa}\right)$, then the continuity of $w$ gives $w\left(\bar{x}_{J}\right)=\kappa$, which easily gives a contradiction with the choice of $\varepsilon,\left(A_{2}\right)$, and (12). Therefore, $\bar{x}_{J} \notin \partial\left(\mathcal{S}_{\kappa}\right)$.

Case 2: If $\bar{x}_{J} \in \mathcal{T}$, then it follows from $S T C_{f}(\mathcal{T})$, hypothesis 4) from the theorem, and standard estimates (cf. [1], Chapter 3) that

$$
w(x) \geq \int_{o}^{t_{\star}} \ell\left(y_{x}(s, \overline{\bar{\alpha}}), \overline{\bar{\alpha}}(s)\right) d s-\varepsilon \geq v(x)-\varepsilon,
$$

where $\overline{\bar{\alpha}}$ is the concatenation of $\bar{\alpha}_{J}\left\lceil\left[0, \sigma_{k-1}\right]\right.$ followed by a suitable $\tilde{\beta} \in \mathcal{A}$ for large enough $k$, and $t_{\star}:=t_{x}(\overline{\bar{\alpha}})<\infty$ (cf. [5] for details), so $w(x) \geq v(x)$, by the arbitrariness of $\varepsilon$.

Case 3: Since Case 1 cannot occur, and since Case 2 gives the desired conclusion, it follows from (13) that we can assume that $\bar{x}_{J} \in \partial\left(B_{J}\right)$.

We may now assume $\bar{\sigma}_{J}<\infty$. (Otherwise, replace $\bar{x}_{J}$ with one of the $x_{k}$ 's for which $\operatorname{dist}\left(x_{k}, \partial\left(B_{J}\right)\right) \leq$ $2^{-J}$ and $w\left(x_{k}\right) \geq \mathcal{L}-1$, and replace $\bar{\sigma}_{J}$ with the corresponding $\sigma_{k-1}$.) Notice that $w\left(\bar{x}_{J}\right)<\kappa$ and $\bar{x}_{J}=y_{x}\left(\bar{\sigma}_{J}, \bar{\alpha}_{J}\right)$. Now repeat this procedure but with the initial value $x$ replaced by $\bar{x}_{J}, \mathcal{S}$ replaced by $\mathcal{S}_{\kappa} \cap B_{J+1}$, and $\varepsilon$ replaced by any positive number $\varepsilon_{1}<\varepsilon / 2 \wedge\left[\kappa-w\left(\bar{x}_{J}\right)\right]$ to get a trajectory for an input $\bar{\alpha}_{J+1}$ starting at $\bar{x}_{J}$ which wlog reaches $\partial\left(B_{J+1}\right)$ at time $\bar{\sigma}_{J+1}<\infty$. If we now concatenate this result with $y_{x}\left(\cdot, \bar{\alpha}_{J}\right)\left\lceil\left[0, \bar{\sigma}_{J}\right]\right.$, then we get a trajectory which coincides with $y_{x}\left(\cdot, \bar{\alpha}_{J}\right)$ on $\left[0, \bar{\sigma}_{J}\right]$ and wlog reaches $\partial\left(B_{J+1}\right)$ at (finite) time $\bar{\sigma}_{J}+\bar{\sigma}_{J+1}$.

This process is repeated, with $\varepsilon$ replaced by any positive number $\varepsilon_{q}<\varepsilon / 2^{q} \wedge\left[\kappa-w\left(\bar{x}_{J+q-1}\right)\right]$ and the starting point $x$ replaced by $\bar{x}_{J+q-1}$ in the $q$ th iteration. We can assume $\bar{\sigma}_{J+q}<\infty$ and that all points $\bar{x}_{J+q}=y_{\bar{x}_{J+q-1}}\left(\bar{\sigma}_{J+q}, \bar{\alpha}_{J+q}\right)$ obtained lie in $\partial\left(B_{J+q}\right)$ for all $q$, by the preceding. Fix $\bar{b} \in A$. Set $\overline{\bar{\sigma}}_{q}=\bar{\sigma}_{J}+\bar{\sigma}_{J+1}+\ldots+\bar{\sigma}_{q}$ and $\bar{s}=\lim \sup _{q} \overline{\bar{\sigma}}_{q}$, and let $\hat{\alpha} \in \mathcal{A}$ be the concatenation

$$
\begin{cases}\bar{\alpha}_{J}(s) & \text { if } 0 \leq s<\overline{\bar{\sigma}}_{J} \\ \bar{\alpha}_{J+1}\left(s-\overline{\bar{\sigma}}_{J}\right) & \text { if } \overline{\bar{\sigma}}_{J} \leq s<\overline{\bar{\sigma}}_{J+1}, \\ \vdots & \\ \bar{\alpha}_{J+q}\left(s-\overline{\bar{\sigma}}_{J+q-1}\right) & \text { if } \overline{\bar{\sigma}}_{J+q-1} \leq s<\overline{\bar{\sigma}}_{J+q}, \\ \vdots & \\ \bar{b} & \text { if } \bar{s} \leq s\end{cases}
$$

A passage to the limit as $k \rightarrow \infty$ in (12) and a summation then gives

$$
w(x) \geq \int_{0}^{\overline{\bar{\sigma}}_{q}} \ell\left(y_{x}(s, \hat{\alpha}), \hat{\alpha}(s)\right) d s+w\left(\bar{x}_{q}\right)-2 \varepsilon
$$

for all $q \geq J$. If $\bar{s}$ is finite, then we get

$$
\begin{aligned}
& \partial\left(B_{J+q+1}\right) \ni y_{\bar{x}_{J+q}}\left(\bar{\sigma}_{J+q+1}, \bar{\alpha}_{J+q+1}\right) \\
& =y_{x}\left(\overline{\bar{\sigma}}_{J+q+1}, \hat{\alpha}\right) \rightarrow y_{x}(\bar{s}, \hat{\alpha}) \text { as } q \rightarrow \infty
\end{aligned}
$$

which contradicts (3) in Definition 2.1. Since $w \in$ $W B B$ and $\bar{x}_{q} \in \partial\left(B_{q}\right)$ for all $q \geq J$, a passage to the limit as $q \rightarrow \infty$ in (14) therefore gives

$$
\int_{0}^{\infty} \ell\left(y_{x}(s, \hat{\alpha}), \hat{\alpha}(s)\right) d s \leq w(x)+\text { constant }
$$


Since $y_{x}\left(\overline{\bar{\sigma}}_{J+q+1}, \hat{\alpha}\right)=y_{\bar{x}_{J+q}}\left(\bar{\sigma}_{J+q+1}, \bar{\alpha}_{J+q+1}\right) \in$ $\partial\left(B_{J+q+1}\right)$ for $q=1,2, \ldots$, we also have

$$
\limsup _{s \rightarrow \infty}\left\|y_{x}(s, \hat{\alpha})\right\|=\infty .
$$

But (15)-(16) stand in contradiction with (8). Consequently, it must be the case that $\bar{x}_{J+q} \in \mathcal{T}$ for large enough $q$. By the argument of Case 2 above with $\bar{x}_{J}$ replaced by $\bar{x}_{J+q} \in \mathcal{T}$, and the arbitrariness of $\varepsilon>0$, this gives the desired inequality $w(x) \geq v(x)$ and completes the proof.

\section{Shifted Fuller Example Revisited}

In this section, we indicate why the perturbed problems in the Shifted Fuller Example, corresponding to the data (7) for all $p \geq 0$, all satisfy the requirements of Theorem 1 (cf. [5] for details). Condition 3 ) holds since (i) the dynamics $f_{p}$ in (7) agrees with the $\mathrm{FE}$ dynamics in a suitable neighborhood of the $y$-axis and the Lagrangians $\ell_{p}$ assign a positive cost to staying at $(0,0)$ when $p>0$ and (ii) the FE satisfies condition 3$)$. Condition $S T C\left(\mathcal{T}_{p}\right)$ holds because $f_{p}(x, a)=\left(x_{2}-p, a\right)$ near $\mathcal{T}_{p}:=\{(p, p)\}$ and the FE satisfies $S T C\{(0,0)\}$ (cf. [4]), along with a change of coordinates. Finally, (8) holds by a variant of Barbălat's Lemma proven in [7]. We conclude as follows:

Corollary 4.1 Let $p \geq 0$, and choose the data (7). Let $v_{p}$ be the value function (5) for these data. If $w \in W B B$ is a continuous solution of

$$
\left[-x_{2}+p \Phi(x, p)\right](D w(x))_{1}+\left|(D w(x))_{2}\right|-x_{1}^{2}=0
$$

on $\mathbb{R}^{2} \backslash \mathcal{T}_{p}$ that satisfies $w(p, p)=0$, then $w \equiv v_{p}$.

Taking $p=0$ in Corollary 4.1 gives the FE uniqueness characterization in [7]. Note that Corollary 4.1 applies to problems violating both of the usual assumptions (4) and (9), and that it establishes uniqueness of solutions of the HJBE in a class of functions which includes functions which are neither proper nor bounded-from-below.

Remark 4.2 Since $x \mapsto x^{2}$ is convex, $v_{o}$ is convex on $\mathbb{R}^{2}$ and therefore continuous. Using the Backward Dynamic Programming Principle (cf. [1]), one shows that $(x, y) \mapsto \tilde{v}(x, y):=-v_{o}(-x, y)$ is also a solution of the Fuller Example HJBE on $\mathbb{R}^{2} \backslash\{0\}$ which is null at the origin (cf. [5]). Note that $\tilde{v} \notin W B B$, since $v_{o}$ is proper (cf. [4]). Therefore, $v_{o}$ is the unique continuous solution $w \in W B B$ of the corresponding HJBE on $\mathbb{R}^{2} \backslash\{0\}$ that is null at the origin, and the parameter choice $p=0$ shows that the $w \in W B B$ hypothesis in the corollary cannot be omitted.

\section{References}

[1] Bardi, M. and I. Capuzzo-Dolcetta, Optimal Control and Viscosity Solutions of HamiltonJacobi-Bellman Equations, Birkhäuser, Boston, 1997.

[2] Bardi, M. and P. Soravia, "Hamilton-Jacobi equations with singular boundary conditions on a free boundary and applications to differential games," Trans. Amer. Math. Soc., 325(1991), pp. 205-229.

[3] Falcone, M. and C. Makridakis, Eds., Numerical methods for viscosity solutions and applications, World Scientific, Singapore, 2001.

[4] Malisoff, M., "Viscosity solutions of the Bellman equation for exit time optimal control problems with vanishing Lagrangians," SIAM J. Control Optim., 40(2002), pp. 1358-1383.

[5] Malisoff, M., "Bounded-from-below solutions of the Hamilton-Jacobi equation for optimal control problems with exit times: vanishing Lagrangians, eikonal equations, and shape-fromshading," NoDEA Nonlinear Differential Equations and Applications, to appear. ${ }^{3}$

[6] Malisoff, M., "Viscosity solutions of the Bellman equation for infinite horizon optimal control problems with negative instantaneous costs," in Proc. 41st IEEE Conference on Decision and Control, Las Vegas, NV, December 2002, to appear. ${ }^{3}$

[7] Malisoff, M. and H. J. Sussmann, "Further results on the Bellman equation for optimal control problems with exit times and nonnegative Lagrangians: The case of Fuller's Problem," in Proc. 39th IEEE Conference on Decision and Control, Sydney, Australia, December 2000, pp. 2308-2310.

[8] Piccoli, B. and H.J. Sussmann, "Regular synthesis and sufficient conditions for optimality," SIAM J. Control Optim., 39(2000), pp. 359-410.

[9] Soravia, P., "Optimality principles and representation formulas for viscosity solutions of Hamilton-Jacobi equations I: Equations of unbounded and degenerate control problems without uniqueness," Adv. Differential Equations, 4(1999), pp. $275-296$.

\footnotetext{
${ }^{3}$ Preprint at www.math.lsu.edu/ malisoff $/$ research.html
} 